\title{
Familiale Solidarität und gesellschaftliche Reproduktion
}

\author{
Franz SCHULTHEIS ${ }^{1}$ \\ Zeppelin Universität Friedrichshafen
}

\begin{abstract}
Die Familie gilt gemeinhin als eine geradezu archetypische Verkörperung von Solidargemeinschaft. Hierbei sollte jedoch nicht in Vergessenheit geraten, dass die Familie nicht nur ein "Hafen in einer herzlosen Welt» ist, sondern zugleich die Rolle einer zentralen Akteurin der gesellschaftlichen Reproduktion von ungleichen Lebenschancen und gesellschaftlichen Hierarchien spielt. Dieses Janusgesicht von Solidarität nach Innen und Selbstbehauptung und Reproduktion von ökonomischen und symbolischen Gütern nach Aussen, das durch Eduard von Hartmann treffend auf den Begriff Familienegoismus gebracht wurde, bringt die elementare Doppeldynamik von gesellschaftlicher Schliessung und Ausschliessung geradezu in Reinform zur Geltung.

Schlüsselbegriffe: Familie, Solidarität, soziale Reproduktion, Kapital
\end{abstract}

\section{Einleitung}

Die Familie gilt gemeinhin als eine geradezu archetypische Verkörperung der Solidargemeinschaft. Hierbei sollte jedoch nicht in Vergessenheit geraten, dass die Familie nicht nur ein «Hafen in einer herzlosen Welt» (Lasch 1976) ist, sondern zugleich die Rolle einer zentralen Akteurin der gesellschaftlichen Reproduktion von ungleichen Lebenschancen und gesellschaftlichen Hierarchien innehat. Dieses Janusgesicht von Solidarität nach Innen (Binnenraum) und Selbstbehauptung und Reproduktion von ökonomischen und symbolischen Gütern nach Aussen, das durch Eduard von Hartmann (1923) treffend auf den Begriff Familienegoismus gebracht wurde, bringt die elementare Doppeldynamik von gesellschaftlicher Schliessung und Ausschliessung (Max Weber 1972: 324) geradezu in Reinform zur Geltung. Die Familie grenzt sich ab, schliesst sich ab für die exklusive Organisation von Solidarität im Innenraum, schafft Anrechte auf Unterstützung für ihre Mitglieder und grenzt dabei in ein- und demselben Schritt Familienexterne von dieser Solidarität aus. Soziale Inklusion und Exklusion gehen Hand in Hand, sind die zwei Seiten des familialen Janusgesichts.

\footnotetext{
${ }^{1}$ Franz Schultheis ist Professor für Soziologie der Kunst und Kreativwirtschaft an der Zeppelin Universität Friedrichshafen (franz.schultheis@zu.de).
}

DOI: http://dx.doi.org/10.18753/2297-8224-176 
Um die Frage gesellschaftlicher Solidarität ranken sich eine Vielzahl von Diskursen, Narrativen und Mythologien. Sie manifestieren sich in einem Feld symbolischer Kämpfe mit beachtlichen ideologischen und politischen enjeux. Die in diesem Diskursfeld engagierten Akteure, vermeintlich geeint durch das geteilte Engagement rund um das Gut Solidarität, stehen dabei nicht einfach nur in einem Verhältnis der Kooperation, sondern auch dem der Konkurrenz zueinander. Hierbei geht es insbesondere um die Deutungshoheit in Sachen legitimer Trägerschaft und berechtigter Adressaten von Solidarität, wie auch um die Frage der Verfügung über die hierbei zu verteilenden Ressourcen. Es geht gewissermassen um die Konkurrenz um das Monopol der legitimen Definition von Solidarität (vgl. hierzu Leitner 2003, 2013). Diese weltanschaulichen Kämpfe haben in der Moderne klare Konturen, um nicht zu sagen Fronten, angenommen, bei denen sich ab dem frühen 19. Jahrhundert mit dem Begriff Familialismus, in der Gegenlage zum Wohlfahrtsstaat im Sinne einer staatlichen Organisation von Solidarität, auch konzeptuelle Waffen im sogenannten Kulturkampf zwischen Kirche und Staat herauskristallisierten. ${ }^{2}$

\section{Familie als Hort urwüchsiger Solidarität}

Ab den 1830er Jahren entsteht mit dem sogenannten Sozialkatholizismus eine bedeutende, international agierende, soziale Bewegung, die in der Familie die Keimzelle von Gesellschaft und Staat schlechthin sieht und ihr hierüber den absoluten Vorrang bei der Daseinsvorsorge und Fürsorge zuspricht. Autoren wie Frédéric Le Play (1806-1882), einer der wichtigsten Protagonisten dieser Bewegung und wohl auch der erste Vertreter der Familiensoziologie schlechthin, idealisieren die Familie als einen Hort naturwüchsiger, spontaner Solidarität, klar abzugrenzen von anderen Anbietern von Solidarität wie staatlichen oder gewerkschaftlichen Institutionen. Die Familie wird in der einschlägigen sozialkatholischen Literatur zur gottgewollten Institution erklärt, die in immer neuen Variationen des gleichen Themas idealtypisch in der Heiligen Familie von Nazareth inkarniert gesehen, verehrt und mit transzendentem Status versehen wird (siehe hierzu Kokorschke 2011). Die Konzeption von Familie als Keimzelle der Gesellschaft transportiert dabei wie selbstverständlich und nicht hinterfragbar Vorstellungen von einem transhistorischen Status und Naturwüchsigkeit und leitet daraus den Anspruch auf Priorität und Höherwertigkeit familialer Solidarität gegenüber anderen gesellschaftlichen Trägern ab. Der idealtypischen Unterscheidung zwischen Gemeinschaft und Gesellschaft im Werk von Ferdinand Tönnies (2010) geht diese dichotome Gegenüberstellung von Familie auf der einen und Staat auf der anderen Seite voraus, bei der der Intimität der interpersonellen Beziehungen zwischen Familienmitgliedern ein Vorrang gegenüber der Anonymität der Massengesellschaft und ihrer bürokratischen Regulierung zugebilligt wird.

Die stark antimodernistischen und fortschrittskritischen Idealisierungen der Keimzelle Familie, gekennzeichnet durch stark nostalgische, rückwärtsgewandte romantische Verbrämungen von der Herd- und Herzenswärme in Zeiten des traditionellen Ganzen Hauses, d.h. eines

\footnotetext{
2 Zur unterschiedlichen historischen Kontextualisierung und Konnotation dieses Begriffs, vgl. z.B. https://www.bpb.de/politik/hintergrund-aktuell/209483/ende-des-kulturkampfes.
} 
stark integrierten Mehrgenerationen-Familienverbandes, rekurrieren nicht zuletzt auf die Idee der Schicksalsgemeinschaft. Seine Familie sucht man nicht aus, man wird in sie hineingeworfen und findet hier die allererste, eindrücklichste und prägendste Erfahrung von gesellschaftlicher Solidarität in «guten wie in schlechten Zeiten». Letzteres hatte im Übrigen schon für die Institution der Ehe zu gelten, die nicht nur nach kirchlichem Recht bis heute, sondern nach dem französischen Code Civil immerhin bis ins späte 19. Jahrhundert als unauflöslich galt. Die ihr zugeschriebene Verpflichtung lebenslanger Solidarität «pour le meilleur et pour le pire» schien im rechtlichen Sonderstatus des Ehevertrags als Statuskontrakt realisiert, der - wie Weber heraushob - den Habitus der beiden Partner grundlegend redefiniert und sich dadurch radikal vom Zweckkontrakt, wie er im kapitalistischen Marktgeschehen vorherrscht, unterscheidet. Hierauf gründet dann auch die Vorstellung von der Unauflösbarkeit der Familienbande und der unabdingbaren Verpflichtung zur wechselseitigen Solidarität, wie sie ja seit der Etablierung des modernen Familienrechts im französischen Code Civil (1804) ja auch bis heute in Gestalt der Unterhaltsverpflichtung festgeschrieben ist.

Die mit dem Familialismus einhergehende Ideologisierung und Mythologisierung der Familie stilisiert sie als eine Bastion einer vormodernen Welt, in der Produktion und Reproduktion unter einem Dach - dem Oikos - harmonisch koexistierten, bevor sie unter der kapitalistischen Wirtschaftsordnung und den damit einhergehenden anonymen Marktlogik, dem Utilitarismus und der Rechenhaftigkeit des Homo oeconomicus auseinandergerissen wurden. Anders formuliert kann man im Familialismus zurecht eine Form konservativer Kapitalismuskritik sehen. Doch diese konservative soziale und ideologische Bewegung hat einen Widerpart, der sich mit ihr historisch zeitgleich zu äussern beginnt; zwar ebenfalls in einer Kritik am modernen Kapitalismus mündend, jedoch unter ganz anderen Vorzeichen.

\section{Familie als Kern patriarchaler Herrschaft}

Frühsozialisten, wie Robert Owen in Grossbritannien, Henri de Saint-Simon oder Charles Fourier in Frankreich, bis hin zu Marx und Engels, kritisierten die Familie als Trägerin des kapitalistischen Besitzindividualismus, als Ort, wo die geschlechtsspezifische Ungleichheit und patriarchale Herrschaft strukturell angelegt ist und sich reproduziert. Im frühen 19. Jahrhundert sprossen vielfältige Gesellschaftsutopien - man denke z.B. an die phalanstères Fouriers - in denen ein Zusammenleben jenseits von Ehe und exklusiven genealogischen Beziehungen als Gegenentwurf zur Familie gesehen wurde.

Die Frage, worauf gesellschaftliche Ordnung und Solidarität basieren sollten - schicksalsmässige genealogische Zugehörigkeit und Verpflichtung hier, freigewählte wechselseitige Übereinkunft und Solidarität dort - begleitete über fast zwei Jahrhunderte die wechselnden ideologischen und gesellschaftspolitischen Debatten in unseren westlichen Zivilisationen und fand vor 50 Jahren in der Rebellion von 1968 einen Höhepunkt, als die Familie ins Zentrum der Gesellschaftskritik rückte und die Forderung nach ihrer Überwindung in den Rufen nach einer radikalen Kulturrevolution an vorderster Stelle stand. Die Familie wurde hier als Trägerin eines patriarchalen, auf Privateigentum basierenden, massive Ungleichheit produzierenden und reproduzierenden kapitalistischen Systems gesehen und denunziert. Die damalig so einflussrei- 
che Antipsychiatrie-Bewegung (siehe z.B. Cooper 1970; Laing und Esterson 1964) diagnostizierte die familiale Lebensform als grundlegend pathologisch und systematisch Schizophreniefördernd. Dies rief wiederum starke konservative Gegenreaktionen auf den Plan, zum Beispiel durch das einflussreiche Sozialwissenschaftler-Ehepaar Berger, die unter Verweis auf die Werte des Liberalismus zur Verteidigung der bürgerlichen Familie aufriefen, oder die Familie als «Hafen in einer herzlosen Welt» (Lasch 1976) thematisierten.

Wenn die Familie seit nun fast zwei Jahrhunderten in immer neu aufflackernden ideologischen Kämpfen idealisiert und glorifiziert oder aber denunziert wird, so finden diese ideologischen Auseinandersetzungen vielfältige Spuren in der Gesellschaftspolitik und den sie begleitenden Debatten. Man denke beispielsweise an die Fragen der familialen Schlüsselgewalt, der Anerkennung nicht-ehelicher Lebensgemeinschaften und Geburten, der ausserhäuslichen Kindererziehung, der Mutterschaftsversicherung oder der Regulierung innerfamilialer Gewalt (vgl. hierzu im Einzelnen: Schultheis 1999).

\section{Welfare Family versus Welfare State}

Kurzum: Der Anspruch auf Vorrangigkeit der Familie in Sachen Solidarität und gesellschaftlichen Zusammenhalts ist seit langem Gegenstand von gesellschaftspolitischen und weltanschaulichen Kämpfen, bei denen religiöse Motive und Interessen gerade auf Seiten der Verteidiger der familialen Ordnung von grossem Gewicht sind. Gerade die katholische Kirche spielte seit dem frühen 19. Jahrhundert nachhaltig die Rolle einer Fürsprecherin der Familie und ihrer Integrität und Autonomie gegenüber staatlichen Eingriffen in ihre Sphäre (siehe hierzu Schultheis 1989). Mit dem in der Sozialenzyklika Quadragesimo Anno aus dem Jahre 1931 formulierten Prinzip der Subsidiarität schuf sie eine bis in die Gegenwart fortwirkende Legitimation für den Anspruch auf Vorrangigkeit familialer Solidarität. Dies ist natürlich auch in dem spezifischen historischen Kontext zu begreifen, da es angesichts erstarkender autoritärer und totalitärer Ideologien darum ging, einem möglichen staatlichen Eingriff in die Privatsphäre der Familie und ihrer relativen Vorrangigkeit bei der Kindererziehung eine Grenzlinie zu ziehen, die der Staat nicht überschreiten sollte. Nach dem Subsidiaritätsprinzip gilt es, die einem hilfsbedürftigen Individuum nahestehendste Gruppe oder Institution als vorrangig zuständig anzusehen - eine Position, die auch in der anti-etatistischen Haltung des Neoliberalismus Entsprechungen findet. Damit ist selbstredend zunächst die Familie gemeint, nachrangig aber auch die unmittelbare gesellschaftliche Umgebung wie Nachbarschaft, Dorf, Kanton und erst zuallerletzt der Zentralstaat. Hiermit wird ein Lokalitätsprinzip der Solidarität etabliert, bei dem die Kirche sozusagen im Dorf bleibt. Die Kirche selbst aber ordnet sich mittels einer weiteren Implikation des Subsidiaritätsprinzips ebenfalls eine Vorrangigkeit gegenüber dem Staat zu. Kirchliche Institutionen organisierten seit dem Mittelalter unter dem Leitmotiv der Caritas massgeblich die Solidarität für die Armutsbevölkerung, die Notleidenden, Kranken und Alten und postulierte mit dem Subsidiaritätsprinzip, dass nicht-staatliche Organisationen in Sachen sozialer Sicherung gegenüber staatlichen Vorrang zu haben hätten.

Doch auch hier waren die symbolischen Kämpfe rund um Solidarität durchaus von handfesten Interessen der beteiligten Institutionen an sich selbst und ihren Einflusssphären motiviert und begleitet. Das kirchliche Engagement als Anwältin der Keimzelle Familie und ihrer 
Autonomie gegenüber staatlichem Einfluss ist auch vor dem Hintergrund des sogenannten Kulturkampfes zwischen Staat und Kirche rund um die Frage der Kindererziehung zu sehen. Durch die Einführung der allgemeinen Schulpflicht in den 1880er Jahren hatte der moderne Staat den in den meisten westlichen Ländern bis dahin weitgehend in kirchlicher Hand gelegenen Zugriff auf die Erziehung der Kinder und damit der Reproduktion ihrer eigenen Klientel an sich gerissen und einen Monopolanspruch darauf erhoben. Gleiches gilt für den modernen Sozialstaat, der fast zeitgleich damit begann, in die bis dahin weitgehend von kirchlichen, aber auch gewerkschaftlichen Organisationen bereitgestellte Solidarität für Notleidende an sich zu reissen. Der Schulterschluss zwischen Kirche und der Keimzelle Familie unter dem Leitmotiv der Subsidiarität stellte demnach auch einen Versuch dar, traditionelle Formen solidarischer Daseinsfürsorge gegen Prozesse der Modernisierung unter staatlichen Auspizien zu verteidigen. Diese Haltung kennzeichnet auch die in liberalen Wohlfahrtsstaaten wie den USA, Grossbritannien oder - bedingt - auch der Schweiz vorherrschende Skepsis gegenüber einem staatlichen Monopolanspruch auf die Daseinssicherung und kontrastiert deutlich mit dem in sozialdemokratischen welfare states wie Schweden historisch herausentwickelten staatszentrierten Modell der sozialen Sicherung.

Der Einsatz für Solidarität scheint also nie ganz und gar selbstlos, sondern bringt immer auch Ansprüche auf Definitions- und Deutungsmacht, gesellschaftliche Einflussmöglichkeiten und Schaffung von Gefolgschaft mit sich. Doch wie ist es um die Bruderethik der familialen Solidarität bestellt? Handelt es sich bei der Familienmoral der wechselseitigen Verpflichtung zu Unterstützung und Schutz um pure Selbstlosigkeit, oder verbirgt sich hier nicht auch, wie anfangs mit dem Konzept des Familienegoismus postuliert, eine Form der Selbstbehauptung und des Strebens nach Besitzstandswahrung bzw. -mehrung mittels spezifischer familialer Reproduktionsstrategien?

\section{Familiale Solidarität als anthropologische Konstante}

Steigt man von den Höhen der weltanschaulichen Familien-Rhetorik hinab zu den vielfältigen empirischen Realitäten familialer Lebensformen und Alltagspraxen, kann man zunächst feststellen: Familien erbringen tagtäglich tausendfach und scheinbar selbstverständlich solidarisches Handeln. Dies geschieht meistens ganz stillschweigend und unhinterfragt, denn das Familienethos beruht ja gerade darauf, dass Eigeninteressen, utilitaristisches Profitdenken und Berechnung wie sie ausserhalb der Familie zum selbstverständlichen Repertoire des rational agierenden Homo oeconomicus gehören, tabuisiert und verbrämt werden. Anders gesagt funktioniert familiale Solidarität nur dann reibungslos, wenn sie von einer geteilten Heuchelei begleitet wird: Egoistische Interessenabwägungen und Handeln in üblichen rational choice-Strategien sind zwar omnipräsent, werden jedoch unter Vorgaben des ethischen Grundprinzips familialer Solidarität, der unhinterfragbaren Verpflichtung zur Gabe (Mauss 1973) verschwiegen. Offenes Zeigen egoistischer Interessen ist im Kontext der Familie in der Regel verpönt - aber auch hier gibt es wohl ausreichend zu beobachtende Ausnahmen, um diese ideale moralische Regel zu bestätigen. In der Familie gilt das Solidaritätsprinzip einer - um es mit einem Konzept Webers zu benennen - Ethik der Brüderlichkeit. Hier gilt die elementare Form sozialen Handelns: Ego schuldet Alter grundsätzlich ein Geben, aus der scheinbar voraussetzungslosen Gabe 
Egos aber resultiert für Alter nach der Ethik der Reziprozität dann die moralische Verpflichtung zur Gegengabe.

Warum aber kann man gerade in der Familie berechtigterweise die Ursprünge einer solch ursprünglichen und elementaren Form der Trägerin von Solidarität sehen? Aus anthropologischer Sicht erscheint der Mensch als Mängelwesen bzw. als biologische Frühgeburt. Eigentlich müsste nach allen biologischen Merkmalen die Schwangerschaft beim Homo sapiens 18 Monate dauern; stattdessen wird das geborene Menschenkind in einem Zustand völliger Hilflosigkeit in die Welt geworfen und landet dann im Schosse einer Familie. Laut Adolf Portmann (1965) übernimmt die Familie die Funktion eines sozialen Uterus und ermöglicht so das Überlebendes Neugeborenen. Im Hinblick auf diese anthropologischen Zusammenhänge kann man wohl ohne Zweifel der Familie einen von allen anderen Formen und Trägern von Solidarität klar abgehobenen Status zubilligen. Die Familie wird angesichts ihrer existentiell unverzichtbaren Funktion für die biologische Reproduktion und zugleich angesichts der extremen Instinktreduktion des menschlichen Mängelwesens auch mit einer beachtlichen Prägekraft bezüglich jeder neuen Generation ausgestattet, denn die für den Menschen kennzeichnende Form der Entwicklung verläuft ganz massgeblich über Prozesse der intergenerationellen Transmission von Kompetenzen aller Art (Sozialisation und Enkulturation).

So kommt es, dass das Kind zunächst notwendigerweise von der Regel direkter Reziprozität ausgenommen ist. Marcel Mauss (1973) hat diesbezüglich von einer indirekten Reziprozität gesprochen. Sie besagt: Tue das, was deine Eltern für Dich getan haben, für deine eigenen Nachkommen. Verbunden damit ist die Idee einer intergenerationellen Gerechtigkeit: Demnach soll man mindestens so viel an die nächste Generation weitervererben, wie man selbst von den eigenen Vorfahren bekommen hat. Diese ethische Grundregel der Familie impliziert auch, dass man den erworbenen Besitzstand für die nachfolgende Generation sichern und das ererbte Patrimonium mindestens im gleichen Umfang tradieren sollte. In diesem Zusammenhang liesse sich mit einem Begriff Emile Durkheims auch von Familienkommunismus sprechen, der auf die besondere Qualität familialer Solidarität gegenüber anderen Formen solidarischer Beziehungen verweist.

Im bisherigen Licht betrachtet könnte aber ein Zerrbild der Familie als geradezu paradiesisch anmutender Hort uneingeschränkter Selbstlosigkeit entstehen, dem nun in einem zweiten Schritt durch Blick auf die Rückseite der Medaille ein anderes Gesicht der Familie als Hauptakteurin gesellschaftlicher Reproduktion entgegenzuhalten ist.

\section{Zur familialen Reproduktion gesellschaftlicher Strukturen}

In Anlehnung an die Soziologie Pierre Bourdieus (1994) können wir dabei von verschiedenen Strategien und Wegen familialer Reproduktion sprechen, bei der unterschiedliche Formen von Kapital (ökonomisches, kulturelles, soziales und symbolisches) von Generation zu Generation vererbt werden.

Wie oben bereits erwähnt, kann das Streben nach Besitzstandswahrung und -mehrung als ein im familialen Ethos angelegtes ethisches Grundprinzip gelten. Bei der intergenerationellen Transmission familialen Eigentums kam es prototypisch in Gestalt des Erstgeborenen-Rechts 
(primogéniture) zur Geltung. Dieses Modell der Vererbung - ein Ausdruck von Familienegoismus par excellence - produzierte systematisch Opfer - nämlich grundsätzlich die weiblichen Nachkommen sowie die jüngeren Brüder - zum Zweck der Vermeidung einer Zersplitterung des agrarischen Familienbesitzes durch Erbteilung. Erst mit dem modernen Zivilrecht wurde vor gut zwei Jahrhunderten das Prinzip der Erbgleichheit, das bis dahin nur in bestimmten Regionen praktiziert wurde, auf breiter Front durchgesetzt. Interessanterweise konnten Demographen dann sehr frühzeitig einen deutlichen Geburtenrückgang in den betroffenen landwirtschaftlichen Familien dokumentieren. Viel spricht dafür, dass hier eine Gegenstrategie zur oktroyierten Erbteilung zur Geltung kam, die wir weiter unten als Fruchtbarkeitsstrategien bezeichnen.

\section{Heiratsstrategien im Dienst familialer Reproduktion}

In den meisten traditionellen Agrargesellschaften waren Ehen nicht einfach eine freie, von Gefühlen zweier Individuen getragene Entscheidung, sondern durch Dritte mittels familialer Strategien über die Köpfe der Betroffenen hinweg entschiedene Vernunftheirat, zweckrational im Dienst der Optimierung von Ressourcen und Lebenschancen ausgehandelt. Natürlich werden in modernen - im Sinne von zeitgenössischen westlichen - Familien die Ehen der Nachkommen nicht mehr angebahnt, geschweige denn von den Eltern oktroyiert. Dies scheint in der Regel aber auch gar nicht notwendig, denn die verfügbaren Daten über die sozialstrukturellen Profile von Paaren, ob verheiratet oder zusammenlebend, verweist auf das Phänomen einer ausgeprägten Homogamie: auch wenn es aus der Sicht von Eltern durchaus noch Mesalliancen geben kann - die Mehrheit der Paarbildungen steht dennoch unter dem Motto des «Gleich und Gleich gesellt sich gern» und trägt somit zur spezifischen Dynamik familialer Reproduktion wie selbstverständlich bei (vgl. hierzu z.B. Ravazzini et al. 2019).

\section{Strategien der Fruchtbarkeit}

Seit gut zwei Jahrhunderten zeigen einschlägige statistische Untersuchungen immer wieder aufs Neue, dass unterschiedliche Populationen - man mag sie als Klassen, Schichten oder soziokulturelle Milieus bezeichnen - oft ein deutlich unterschiedliches Fruchtbarkeitsverhalten mit entsprechenden Konsequenzen für die jeweilige Natalität an den Tag legen. Dies setzte ein mit dem oben genannten Geburtenrückgang bei bäuerlichen Familien nach der Einführung der Erbgleichheit. Seit dem späten 19. Jahrhundert lässt sich zeigen, dass die Geburtenstatistiken der industriellen Gesellschaften eine U-Form aufweisen und hohe Geburtenraten bei den Armen und wenig Privilegierten mit hoher Natalität bei den privilegierten Familien einhergeht, während man eine im Durchschnitt deutlich niedrigere Kinderzahl bei Mittelschicht-Familien antrifft (vgl. Bourdieu 1966). Aus soziologischer Sicht lässt sich hier postulieren, dass der Wunsch nach sozialem Aufstieg, der ein zentrales Motiv der mittels Bildungsanstrengungen aufstrebenden Mittelschichten repräsentiert, sich darin äussert, dass man mittels Geburtenplanung versucht, die Familienlasten so niedrig zu halten, dass man einem oder maximal zwei Kindern qua Investition in den Bildungsweg die Möglichkeit eines intergenerationellen sozialen Aufstiegs 
verschafft. Überhaupt ist die familiale Investition in Bildungskapital als eine Sonderform kulturellen Kapitals ein vorrangiges Terrain familialer Reproduktionsstrategien. Während familiale Reproduktion in bäuerlichen Familien durch das Motiv der Besitzstandswahrung beziehungsweise -mehrung strategisch orientiert wird und in bürgerlichen Unternehmer-Familien durch jenes der Wahrung eines erreichten Niveaus der Akkumulation ökonomischen Kapitals, sind die modernen Mittelschichten - auch im Kontrast zu ihren traditionellen Vorläufern wie der Handwerkerschaft - in ihrem sozialen Status und ihrer beruflichen Praxis primär über die Verfügung über kulturelles Kapital bestimmt und orientieren sich in ihren Reproduktionsstrategien hauptsächlich an dessen Tradierung.

\section{Familiale Transmission kulturellen Kapitals}

Auf die besondere Prägekraft familialer Sozialisation wurde bereits oben eingegangen. Sie schöpft ihre besondere Nachhaltigkeit für die Persönlichkeitsentwicklung durch die schon in der frühesten Kindheit einsetzende Enkulturation mittels vielfältigster Lernprozesse. Die Familie ist ein Hort der kulturellen Transmission aller erdenklichen mentalen, moralischen, ästhetischen und verhaltensmässigen Dispositionen, welche durch zumeist un- oder zumindest vorbewusste Übernahme vorgelebter Praktiken im familialen Kontext schrittweise verinnerlicht bzw. zu einem Habitus einverleibt werden. Hierzu zählen scheinbar banale Tatbestände wie unterschiedliche Körpertechniken - wie man sitzt, schläft, läuft etc. ist jeweils kulturell spezifisch und wird im familialen Kontext wie selbstverständlich erlernt. Weiterhin die Art, wie und was man isst, redet, sich benimmt: der gesamte basale code of conduct einer Kultur wird primär, und deshalb tief einprägsam, im Schosse der Familie übermittelt. Diese kulturellen Muster sind jedoch je nach sozialer Klasse oder Schicht hochgradig variabel. Je nach Bildungsnähe beziehungsweise -ferne und den damit verfügbaren Ressourcen an kulturellem Kapital der Eltern werden Kindern aus unterschiedlichen Kontexten schon sehr frühzeitig mit mehr oder weniger legitim erscheinenden kulturellen Dispositionen ausgestattet, d.h. mit mehr oder weniger elaborierten Sprachvermögen, Beherrschung von Benimmregeln, Fähigkeiten der Selbstkontrolle und -disziplin etc., Kompetenzen, die sich auf dem späteren Weg durch Bildungsinstitutionen und Berufslaufbahnen in divergierenden Chancen auf Erfolg niederschlagen (vgl. Bourdieu 1994). Anders gesagt ist die bis heute andauernde soziale Frage der massiven Chancenungleichheit im Bildungswesen nicht primär dessen Selektionsmechanismen anzulasten, sondern es sind die Weichen, die schon im Vorfeld der schulischen Erziehung durch die Familie und ihre Vererbung kulturellen Kapitals gestellt werden.

Es liessen sich darüber hinaus noch eine Vielzahl weiterer Aspekte der enormen Bedeutung familialer Reproduktionsprozesse beleuchten; man denke z.B. an die Weitergabe symbolischen Kapitals in Gestalt des guten Namens bzw. Rufs der Familie - ihre Anerkennung und Reputation - oder die intergenrationelle Transmission des von der Familie akkumulierten Sozialkapitals, d.h. die vorhandenen Netzwerke potentieller Unterstützung und Förderung, die ebenfalls eine gesellschaftlich stark ungleich verteilte Ressource für Lebenschancen (Weber 1972) darstellt. 


\section{Fazit}

Die Familie repräsentiert zweifellos eine archetypische Form von Solidargemeinschaft. Mit ihr wird der Wert solidarischen Handels so selbstverständlich assoziiert, dass man sich auch in anderen sozialen Kontexten, wie Unternehmungen oder Vereinen, Parteien oder religiösen Gruppen, ihres Namens bedient, um die beanspruchte eigene, starke soziale Inklusion zu symbolisieren. Mit der paradigmatischen und emblematischen Sozialfigur Familie aber geht eben immer zugleich auch eine Dynamik der Exklusion Dritter wie auch Manifestationen korporatistischer Interessen bzw. Egoismen einher. Die Familie ist eine besonders geschlossene Solidargemeinschaft: nach innen das Prinzip selbstverständlicher wechselseitiger moralischer Verpflichtung zum Geben ohne Berechnung, nach aussen hin aber konkurrenzorientiert beim Streben nach optimalen Durchsetzungschancen für die Nachkommen und in Bezug auf Besitzstandswahrung bzw. -mehrung hinsichtlich der gegebenen Ressourcen an materiellen, kulturellen und symbolischen Gütern. Friedrich Engels' Kritik an der bürgerlichen Familie als Hort und Bewahrerin von Privateigentum wie auch einer patriarchalen Herrschaft ebenso wie Pierre Bourdieus Analysen der enormen Macht von Familie hinsichtlich der Reproduktion gesellschaftlicher Ungleichheit beim Zugang zu Lebenschancen aller Art sind heute angesichts der sich immer weiter öffnenden Schere bei der Verteilung von Vermögen und Einkommen weiterhin von Aktualität und Relevanz. Ihre Perspektiven auf die janusgesichtige Rolle und Funktion von Familie - nach innen solidarisch, nach aussen egoistisch und berechnend - verdeutlichen, dass das vermeintlich Private in Wirklichkeit durch und durch politisch ist. Aus diesem Grund sind politische Auseinandersetzungen rund um Familie von Fragen wie Ehe- oder Erbrecht, über Kindergeld und Steuerfreibeträge, bis hin zu Debatten über die Gleichstellung gleichgeschlechtlicher Paare und einem Adoptionsrecht für sie, nie einfach nur isolierte zivil- oder sozialrechtliche Detailfragen, sondern haben gesellschaftspolitischen Charakter insofern sie jeweils ordnungspolitische Implikationen aufweisen. Deshalb bedeutet auch die Beanspruchung von Deutungshoheit über Familienangelegenheiten immer auch einen Anspruch auf die legitime Auslegung normativer Grundlagen gesellschaftlicher Ordnung. Anders und mit dem Titel eines gemeinsam mit Francois de Singly 1991 herausgegebenen Tagungsbandes gesagt, sind Affaires de Famille immer zutiefst Affaires d'Etat.

\section{Danksagung}

Der Autor bedankt sich bei den anonymen GutachterInnen und der Redaktion des Journals sozialpolitik.ch für die wertvollen Hinweise und Kommentare.

\section{Deklaration von Interessenkonflikten}

Der Autor deklariert keine Interessenkonflikte in Bezug auf Forschung, Autorenschaft und Publikation des Artikels. 


\section{Finanzierung}

Der Autor hat keine finanzielle Unterstützung für die Forschung, die Autorenschaft und die Publikation dieses Artikels erhalten.

\section{Literaturverzeichnis}

Berger, Brigitte und Peter Berger (1984). In Verteidigung der bürgerlichen Familie. Frankfurt: Fischer.

Bourdieu, Pierre (1994). Stratégies de reproduction et modes de domination, Actes de la recherche en sciences sociales, 105, 3-12.

Bourdieu, Pierre (1966). La fin d'un malthusianisme. In: Bourdieu, Pierre et al: Le Partage des bénéfices: expansion et inégalités en France. Paris: Editions de minuit, 136-154.

Cooper, David (1970). Psychiatrie et anti-psychiatrie. Paris: Ed. du Seuil.

Engels, Friedrich (1946). Der Ursprung der Familie, des Privateigentums und des Staats. Berlin: Verlag Neuer Weg.

Hartmann, Eduard von (1923). Gedanken über den Staat, Politik und Sozialismus. Leipzig, Alfred Kröner.

Kokorschke, Albrecht (2011). Die Heilige Familie und ihre Folgen. Frankfurt: Fischer.

Laing, Ronald D. und Aaron Esterson (1964). Sanity, Madness and the Family. London: Penguin Books.

Lasch, Christopher (1976). The Family as a Haven in a Heartless World, Salmagundi 35 (Fall): 42-55.

Leitner, Sigrid (2003). Varieties of familialism. The caring function of the family in comparative perspective, European Societies 5(4): 353-375.

Leitner, Sigrid (2013). Varianten von Familialismus. Eine historisch vergleichende Analyse der Kinderbetreuungs- und Altenpflegepolitiken in kontinentaleuropäischen Wohlfahrtsstaaten. Berlin: Dunckler und Humblot.

Le Play, Frederique (1871). L'organisation de la famille. Paris.

Mauss, Marcel (1973). Essai sur le don. Forme et raison de l'échange dans les sociétés archaïques. Sociologie et Anthropologie. Paris, 149-279.

Portmann, Adolf (1965). Aufbruch der Lebensforschung. Zürich: Rhein Verlag.

Ravazzini, Laura, Ursina Kuhn und Christian Suter (2019). Gegensätze ziehen sich nicht an - die Rolle von Bildung und Einkommen bei der Paarbildung in der Schweiz, Social Change in Switzerland, 17, zuletzt eingesehen am 05.05.2021 unter: https://www.socialchangeswitzerland.ch/?p=1705.

Schultheis, Franz (1988). Sozialgeschichte der französischen Familienpolitik. Frankfurt: Campus.

Schultheis, Franz und François de Singly (1991) (Hrsg.). Affaires de Familles - Affaires d'Etat. Nancy: Edition de l'est.

Schultheis, Franz (1999). Familien und Politik. Konstanz: Universitätsverlag Konstanz.

Tönnies, Ferdinand (2010). Gemeinschaft und Gesellschaft. Grundbegriffe der reinen Soziologie. Darmstadt: WBG-Verlag. 
Weber, Max (1973). Wirtschaft und Gesellschaft. Tübingen: Mohr Siebeck. 